\title{
Palliative home-based technology from a practitioner's perspective: benefits and disadvantages
}

This article was published in the following Dove Press journal:

Smart Homecare Technology and TeleHealth

18 November 2014

Number of times this article has been viewed

\section{Bridget $M$ Johnston}

Sue Ryder Care Centre for the Study of Supportive, Palliative, and End of Life Care, School of Health Sciences, University of Nottingham, Queen's Medical Centre, Nottingham, UK
Correspondence: Bridget M Johnston Sue Ryder Care Centre for the Study of Supportive, Palliative, and End of Life Care, School of Health Sciences, University of Nottingham, Queen's Medical Centre, Derby Road, Nottingham NG7 2HA, UK

Email bridget.johnston@nottingham.ac.uk

\begin{abstract}
This critical review paper explores the concept of palliative home-based technology from a practitioner's perspective. The aim of the critical review was to scope information available from published and unpublished research on the current state of palliative home-based technology, practitioner-focused perspectives, patient-focused perspectives, quality of life, and the implications for clinical practice. Published and unpublished studies were included. An example of one UK patient-centered home-based technology is explored as an exemplar. The evidence suggests that despite the challenges, there are numerous examples of good practice in relation to palliative home-based technology. Improvements in technology mean that telehealth has much to offer people being cared for at home with palliative needs. However, some of the evaluative evidence is limited, and further rigor is needed when evaluating future technologybased solutions innovations.
\end{abstract}

Keywords: technology, telehealth, telemedicine, information technology, palliative care, hospice, terminal illness

\section{Introduction to palliative home care technology Methodology and structure of the paper}

This paper is not intended to be a systematic literature review. The purpose is a discussion paper based upon secondary sources that intends to flag a series of issues around telehealth and palliative care. Our goal is therefore to encourage a critical reflection on the underlying assumptions and the kinds of evidence being used to design and test interventions related to palliative home care technology. It is therefore a critical review with the aim of exploring the potential of telehealth applications to improve quality of life for people with palliative care needs. The following areas will be explored: definitions of palliative care and telehealth; review of studies, including any comparative studies; and practitioner-focused perspectives.

\section{Methods of review}

Literature was identified by electronic searching of the health service-related databases MEDLINE, CINAHL, and Social Sciences Citation Index using broad search terms and was supplemented by a Google Scholar search, hand searching, and a search of the gray literature (using the Google search engine). Research articles were eligible for inclusion in the review if they were written in English and were peer-reviewed papers, if they pertained to adults, or relatives or carers, with palliative care needs and health professionals, and if they were specific to the UK. 
All database searches ran from 1999 to 2014, as it was believed that most relevant studies would be found within this period. Searches of relevant websites were also conducted to identify "gray literature", which includes documents such as unpublished reports, dissertations, articles in obscure journals or journals that are not widely available, some online journals, conference abstracts, policy documents, and technical reports. Although notoriously difficult to identify and retrieve, the author believed that the inclusion of unpublished work was important for a critical review such as this, in order to help minimize the effects of publication bias and to ensure the validity and accuracy of the findings presented.

\section{Definition of palliative care}

"Palliate" has its origins in Medieval Latin palliativus, from the verb palliare, meaning to cloak. Palliative care is one of the fastest-growing specializations in health care worldwide. ${ }^{1,2}$ Palliative care has been associated with total, active, holistic, and therapeutic intervention/s focusing on the quality of life for the patient and his/her family. ${ }^{2,3}$ The universal worldwide accepted definition is that proffered by the World Health Organization. ${ }^{4}$ It stated:

Palliative care is an approach that improves the quality of life of patients and their families facing the problem associated with life-threatening illness, through the prevention and relief of suffering $[\ldots]{ }^{4}$

\section{Definition of telehealth}

The term given to the remote monitoring of patients through information and communication technology is "telecare" or "telehealth" or "telemedicine". 5 The term "telehealth" incorporates a variety of technological devices, including mobile phones, laptop computers, cameras, and remote monitors. Telehealth solutions offer a wide range of functions, including health advice or education via telephone, Skype, or video conferencing, to assess, screen, or monitor disease. Technological solutions can be applied across all health care settings, permitting increased self-care and self-management for patients, as well as helping health care professionals (HCPs) to identify deteriorating symptoms, address and manage these symptoms, and, ultimately, prevent unnecessary hospital or hospice admissions for crisis intervention. ${ }^{6}$

An increasing number of patients are being cared for at home. ${ }^{7}$ One of the health service's main objectives globally is to involve patients in their own care. Discovering new ways to accommodate, maintain, and support self-care and person-centered care is therefore required.
The use of telehealth can help to empower people with palliative care needs, as well as their families, by expediting the provision of real-time communication between patients and HCPs, wherever the patient and their HCPs are located. This can be useful to help transitions within health care from the acute setting to the home setting. Moreover, technical advances in information technology apparatus and solutions, alongside pressures from society, have stimulated both interest in and use of a wide range of telecare and telehealth solutions. Technological solutions can also be cost-effective by rationalizing services based on patient need.

Progress has been made in recent years to confront traditional barriers to the proliferation and uptake of telehealth. ${ }^{8,9}$ Nevertheless, despite advances in technology, telemedicine, and telehealth, the acceptance of many applications has been slow. Also, not all solutions have come from a sound empirical evidence base or have been evaluated effectively. ${ }^{10}$ The potential of telehealth applications to improve quality of life for people with palliative care needs will therefore be explored in this paper.

\section{Review of studies, including any comparative studies Telehealth and palliative/end-of-life care}

Most people with an advanced illness identify being cared for and dying at home as their preferred option. ${ }^{11}$ Moreover, many people report they are fearful of dying in hospital. ${ }^{12}$ Hospital deaths, however, remain prevalent worldwide. ${ }^{13}$

Several studies have explored the issues that impact on keeping people with advanced illness at home. For instance, a systematic review by Gomes and Higginson ${ }^{14}$ identified 17 issues affecting the place of death. These include the illness trajectory, patients' preferences, level of available home care and social support, and the presence or not of informal carers. The authors indicate the need for ongoing evaluation of global policy initiatives that affect place of death.

Patients with palliative care needs who are at home are often unsure how and whom to contact when they experience problems. ${ }^{15}$ Telehealth solutions could be an answer, therefore, to assess and monitor people at home, as well as provide advice, support, and encourage self-management, where appropriate.

There are several telehealth applications that have been reported in palliative care in recent years. ${ }^{16-25}$ Nevertheless, informatics to date has played only a minor role in the evolution of palliative care as a speciality.

On the other hand, it is envisaged that the use of telehealth can empower individuals experiencing life-limiting illnesses, 
as well as their carers, ${ }^{26-28}$ complementing current transitions within health care from the acute to the home setting and rationalizing services based on patient need. When using technology and telehealth solutions, there can, however, be a tension between surveillance and support.

Handheld information technology devices have been in use for some time in health care in solutions as diverse as patient monitoring, simple diagnostic testing, patient tracking, accessing medical literature, and e-prescribing. ${ }^{29-33}$

Telehealth has been shown to be effective in improving symptom management and the experience of care in patients with advanced illness. It is, however, essential that these solutions provide a bridge between the patient and the specialist center without reducing local care or introducing insidious problems by depersonalizing instead of improving personfocused care. It is also necessary that the use of telehealth solutions is needs-driven and that there is commitment to providing long-term solutions. This obligation requires the provision of effective communication pathways, training, and technical support, as well as practical protocols.

\section{Practitioner-focused perspectives}

Videoconferencing services have been used to support multidisciplinary team meetings, including associated imaging and presentation facilities. ${ }^{18,34-38}$ As an example, a new patient administration system was introduced into a London hospice in the UK. This was supplemented by individual operation manuals and one-on-one training sessions for all staff. The system was reported as providing excellent support for multidisciplinary team working. This then led to improved continuity of patient care between community and inpatient staff, improved patient and carer inquiries and out-of-hours care, as well as significantly improved accuracy and readability of information. ${ }^{39}$ It should be noted, however, that this should be perceived as anecdotal evidence until a rigorous empirical evaluation is carried out.

The use of videoconferencing facilities for supporting remote project group meetings has also been reported as part of the Palliative Care Telemedicine Network developed as a partnership between hospital, hospice, charity, and telemedicine services in the UK. The system, in particular, has been identified as a means of overcoming the challenges of delivering palliative care services to patients in rural areas and improving communication between different health care settings. ${ }^{40}$

In a Canadian study, Watanabe et $\mathrm{al}^{22}$ demonstrated that the delivery of specialist multidisciplinary palliative care consultation by videoconferencing is feasible and can improve symptoms, as well as result in cost savings for patients and families, and was acceptable to the HCPs.

Another example is a telemedicine project allowing hospice-based clinical nurse specialists in South Wales to access advice and support on symptom control, disease progression, and pharmacology ${ }^{41}$ by means of a videolink from a consultant in palliative medicine based in England. In addition, the teleconferences were used to discuss issues raised in routine practice that were important to review in order that skills and expertise could be enhanced. The project has shown that telemedicine can provide a useful, possibly cost- and time-effective, addition to current hospice services. Telephone out-of-hours service have also been used, either instead of, or as an adjunct to, existing palliative care services. ${ }^{42-44}$

A systematic review of issues related to palliative care communication and telecare practice discovered that this has three dimensions. ${ }^{23}$ Firstly, to reach high-quality care, HCPs have to build rapport with patients and family caregivers. If patients and their families can trust their care providers and vice versa, experiences of serious illness are more easily shared. Secondly, HCPs need to be focused on the patients' environments and daily lives in order to provide attuned and holistic care. Thirdly, HCPs should be connected continuously to one another to improve team communication, including mutual support and advice.

\section{Patient-focused perspectives}

The model of the "e-hospice" or virtual hospice has been shown to be of benefit to patients and carers. ${ }^{45-47} \mathrm{~A}$ virtual hospice provides support and personalized information about palliative and end-of-life care to patients, family members, HCPs, researchers, and educators in a web-based format. One example is the Canadian Virtual Hospice (http://www. virtualhospice.ca/). ${ }^{47}$ The Canadian Virtual Hospice went online in February 2004 with evidence-based information and an e-health pioneering feature called "Ask a Professional".

The Canadian Virtual Hospice is "A place where: People without ready access to palliative care specialists could ask questions of a clinical team specialized in palliative care". ${ }^{48}$ Patients and family members, as well as HCPs, can access information about all aspects of palliative care. Researchers can also disseminate their findings to allow HCPs to find out about recent developments. ${ }^{48}$

E-hospice, based in the UK, is a globally run news and information resource that brings global news, commentary, and analysis from the world of hospice, palliative, and end-of-life care. Aimed at anyone with an interest in palliative 
care, it offers a single point of access to good practice from around the world. ${ }^{49}$

The benefits of the e-hospice are that patients and family members living remotely can access palliative, end-of-life, and bereavement information and support. Indeed, many people with palliative care needs prefer anonymous support. ${ }^{50}$ Patients and carers have indicated that they benefit from this virtual support. ${ }^{51,52}$

The challenges are that people without internet access or who are not information technology literate cannot access this type of virtual support. ${ }^{46}$

In terms of technological solutions, screening for health problems in palliative care patients and monitoring of disease- or treatment-related symptoms have been reported. Studies have found computer-based screening tools useful in palliative care both in terms of identifying symptoms and when compared with standard paper-based screening tools. ${ }^{25,53,54}$ Conflicts between "high tech" and "high touch" were, however, identified. It should be noted that when the implementation process is conducted with the HCPs involved, benefits of the tool can be accomplished.

More significantly, several studies have consistently reported positive patient experiences in the use of technology. In addition, there is increasing evidence of improved communication between HCPs and patients with the use of telehealth, which can facilitate better continuity of care. ${ }^{33,55,56}$

Moreover, several studies have provided information on the use of dedicated support and advice telephone lines to patients, their carers, and HCPs. ${ }^{57,58}$ One example involves patients registering with the out-of-hours telephone service and naming friends or relatives to whom a dedicated telephone number can be given. Out-of-hours primary care GPs and community nurses requiring information on individual patients and specialist help with symptom problems can also access the service. ${ }^{59}$

Nevertheless, palliative care is dominated by and arguably enhanced by face-to-face communication. This ideal of proximity is reflected in the importance of conversation and touch. ${ }^{23}$ This does, therefore, question whether care that emphasizes touch and face-to-face communication can assimilate technology-mediated communication in the future.

\section{Exemplar of one home-based palliative care technological solution}

An example of a home-based palliative care technological solution was developed by McCall et al ${ }^{19}$ and Johnston et al. ${ }^{53}$ The aim of the trial was to test a mobile phone-based symptom monitoring device for the management of symptoms in patients receiving palliative care at home and assess the feasibility and acceptability of the system in practice. The system, Advanced Symptoms Management System (ASyMS), was developed by Kearney et $\mathrm{al}^{33}$ as an innovative, nurse-led initiative to improve patient outcomes in a technological solution. The system was tested with a purposive sample of 21 patients with palliative care needs and HCPs caring for them in two areas of Scotland. Throughout 1 month of care, patients completed the symptom questionnaire using a mobile phone. This "real-time" symptom information was sent to a secure server. A risk model was incorporated into the system to identify reports of concern over symptoms. The patients who consented to participate in the study were given a mobile phone, a patient diary, and an information booklet. Throughout 1 month of care (once a day), patients completed the symptom questionnaire using the mobile phone (taking 10-15 minutes). This "real-time" symptom information was then automatically sent via a secure general packet radio service connection to the study server. A designated HCP at each site was alerted via a pager of any symptoms causing concern. In this way, severe symptoms were promptly identified and appropriately managed. An alerting system was developed based on a risk model using a "traffic light system" that alerts an HCP carrying a pager to problems the patient is experiencing. An alerting system has not been previously used with palliative care patients living at home.

After completing the symptom questionnaire, patients were immediately provided with tailored self-care information directly relating to the severity of the symptoms reported. They were also able to view their symptom history as graphs on their mobile phone and/or secure web page at any time.

This study was the first of its kind to explore the use of mobile phone technology in the remote monitoring and assessment of symptoms in patients with palliative care needs. Although the study sample was small, the findings provide evidence to suggest that the use of this type of technology is feasible and acceptable to patients with palliative care needs, even those who are very ill and nearing the end of life, as well as the HCPs caring for them. This is particularly supported by the universally positive perceptions expressed by patients using the ASyMSPalliative system, particularly the real-time reporting of symptoms, the self-care advice, and the perceived enhanced communication with HCPs. Patients also expressed feelings of reassurance at the thought of HCPs monitoring their symptoms whilst they were at home. 
The resource and workload implications of the use of ASyMS-Palliative in clinical practice were, however, raised by HCPs, such as the time taken to log patients onto the system and for HCPs to deal with alerts. In addition, using the phone allowed some participants to honestly report their symptoms. This illustrates that some patients may be downplaying their symptoms to prevent distress to their partners and the challenges that are experienced between couples at this time point. One use of the mobile phone could be that it facilitates the exchange of symptom information for patients in a private and confidential way to their palliative care team.

A component of the system that both patients and HCPs found useful, particularly the patients, was the automated evidence-based self-care. HCPs responding to the poststudy questionnaires almost totally agreed that the handset was helpful in monitoring the symptoms of patients receiving palliative care. However, there was less support for the use of the handset to record symptoms. The evaluation of HCPs' overall experience working with ASyMS-Palliative yielded some very important results, with the majority of HCPs finding the system to be time-consuming.

\section{Quality of life}

Quality of life is a commonly measured domain to assess the effectiveness of an intervention. A few studies have examined the effect of home telehealth on quality of life for patients and carers at home. ${ }^{37,51,60,61}$ For instance, Demiris et al, ${ }^{51}$ evaluating their telehealth solution, found that while anxiety scores did decrease, quality of life scores were not significantly changed. Also, Oliver et $\mathrm{al}^{37}$ examined the effect on carer quality of life when using videophones to include carers in team meetings. They found no statistically significant differences between the intervention and control group. Nevertheless, caregivers and HCPs reported that the intervention enriched their relationship. Hebert et a ${ }^{61}$ found that quality of life was similar for patients randomized to receive video visits compared with usual care. Overall, these studies demonstrate that quality of life may be affected positively and that no detrimental effects from the use of telehealth were observed, although more evidence is needed in this area.

Moreover, few studies have explored cost-effectiveness as a consideration of telehealth and palliative care. However, Doolittle et $\mathrm{al}^{62}$ found there were significant savings to be made if videoconferencing was used instead of home visiting. This was concurred by Maudlin et al, ${ }^{21}$ who also reported cost benefits associated with videoconferencing and text messaging when used to prompt and educate patients regarding self-care strategies. They also indicated a reduction in admissions to hospitals, which they attributed to the telehealth initiatives.

However, it is necessary to acknowledge the economic limitations of telehealth in cases where services supplement, rather than replace, visits. ${ }^{63}$ Indeed, it may be found that providing telehealth services actually increases rather than reduces costs for HCPs.

\section{Implications for patient care}

Many of the applications reported here demonstrate real promise in impacting upon patient and carer experience, clinical practice, and health service delivery. In many cases, however, studies are described in isolation from existing systems of care, and little is known about how these would link up and whether this is possible. Perhaps this simply reflects the stage of home-based technology within palliative care and the notion that, although gaining momentum, this is still a relatively new concept.

Linking telehealth to practice depends, however, on HCPs and policymakers working together, as well as organizational structural development. Likewise, integration at the $\mathrm{HCP}$ knowledge and practice level requires the development of new procedures and protocols. ${ }^{27}$

This is echoed in an ethnographic examination of seven telehealth evaluation projects across the $\mathrm{UK},{ }^{64}$ which argues that telehealth has not yet made a way into practice in any systematic fashion. Also, the problems that can happen when evaluation and development of a telehealth service are suggested are often underestimated. They argue that more realistic approaches to evaluation, using mixed methods, are required to improve the relevance of telehealth for service provision within the health care systems so that a workable and practical system can be achieved. ${ }^{43}$

Other concerns from HCPs included issues about the legal status of telehealth, fear that the use of telehealth had added to their workload by disrupting normal practice, concern that the process was different from the protocol, and concern that misdiagnosis might be higher using telemedicine. ${ }^{64}$

In linking telehealth to practice, there are important issues that should be taken into account, such as identifying existing clinical knowledge and practices and anticipating the ways in which these will be changed in incorporating the new technology. The community at which the process or system is aimed should also be ready to accept telehealth. The organizations and HCPs need to be aware of the advantages and disadvantages that might occur for patients, their carers, and HCPs. 
Problems that have been encountered whilst setting up and using telehealth include reception and connectivity issues. ${ }^{59}$ There are also ethical challenges to take into account when linking telehealth technologies to palliative care practice. This includes the privacy and confidentiality of patient data, informed consent, dependency versus independence, equal access issues, lack of the "human touch", and the medicalization of the home environment.

However, there are also several advantages and disadvantages of telemedicine for patients, clinicians, and HCPs. Advantages include improved access to HCPs and decreased time loss and costs for patients, optimized time use and increased productivity for HCPs, and improved service efficiency for providers. ${ }^{65-67}$ On the negative side, the service becomes depersonalized for both patients and clinicians, confidentiality issues may arise, and potential legal implications, including clinical risk management, for HCPs may be a concern. Clinical staff may be required to perform additional tasks such as data collection, which might not be considered the main objective when combined with the pressures of providing a clinical service. ${ }^{67}$

There is also the issue of how to generate and maintain a trustful relationship with HCPs through telecare technologies. These technologies could contribute to trustful relationships by enabling care communication at a distance, thereby extending HCPs' capacities to connect to patients' and proxies' daily social lives outside the usual institutionalized care setting. It may seem easier to establish long-term commitments through telecare, as HCPs and patients are, in principle, no longer bound to particular places and are therefore more flexible in finding time for each other.

In palliative care, adoption of a medical technical paradigm may lead to avoidance of empathic contact. A stereotypical belief about telecare technologies is that they focus on the technical instead of the personal, resulting in objectification instead of humanization. Telecare technologies, like all technologies, are known for their capacity to enlarge some aspects of life while obscuring others.

Nonetheless, it appears that the benefits associated with adopting the further application of telehealth are, despite challenges, both feasible and, with appropriate resources and support form policymakers, organizations, service providers, and the wider health service, achievable.

If telehealth is to play a role in hospice and palliative care, more research is needed to explore the appropriate ways of designing and implementing information systems in this domain and to determine the technology's impact on patient clinical outcomes and the caregiving experience.
An interdisciplinary approach will ensure that we move beyond silos of expertise and design systems driven not by the technological advances but rather by the needs of the stakeholders.

\section{Disclosure}

The author reports no conflicts of interest in this work.

\section{References}

1. Scottish Government. Living and Dying Well: A National Action Plan for Palliative and End of Life Care in Scotland. Edinburgh: Scottish Government; 2008

2. Martin-Moreno J, Harris M, Gorgojo L, Clark D, Normand C, Carlos C. Palliative Care in the European Union. European Parliament Economic and Scientific Policy Department; 2008.

3. Ahmedzai S, Costa A, Blengini $\mathrm{C}$, et al. A new international framework for palliative care. Eur J Cancer. 2004;40(15):2192-2200.

4. World Health Organization. WHO definition of palliative care [web page on the Internet]. Available from: http://www.who.int/cancer/palliative/ definition/en/. Accessed September 9, 2014.

5. Brown S. Next generation telecare and its role in primary and community care. Health Soc Care Community. 2003;11(6):4.

6. Hughes R. Clinical practice in a computer world: considering the issues. J Adv Nurs. 2003;42(4):340-346.

7. Scottish Government. Better Health, Better Care: Action Plan. Edinburgh: Scottish Government; 2007

8. Weinstein RS, Lopez AM, Joseph BA, et al. Telemedicine, telehealth, and mobile health applications that work: opportunities and barriers. Am J Med. 2014;127(3):183-187.

9. Smith AC. Telemedicine: challenges and opportunities. Expert Rev Med Devices. 2007;4(1):5-7.

10. Smith AC, Gray LC. Telemedicine across the ages. Med J Aust. 2009;190(1):15-19.

11. Higginson I. Priorities and Preferences for End of Life Care in England, Wales and Scotland. London, UK: National Council for Hospice and Specialist Palliative Care Services; 2003.

12. Dying Matters Coalition. ComRes Survey on Attitudes Towards Dying, Death and Bereavement Commissioned by the Dying Matters Coalition; 2011.

13. Gomes B, Calanzani N, Higginson IJ. Reversal of the British trends in place of death: time series analysis 2004-2010. Palliat Med. 2012;26(2):102-107.

14. Gomes B, Higginson IJ. Where people die (1974-2030): past trends, future projections and implications for care. Palliat Med. 2008;22(1):33.

15. Ekfors H, Petersson K. A qualitative study of the experiences during radiotherapy of Swedish patients suffering from lung cancer. Oncol Nurs Forum. 2004;31(2):329-334.

16. Freir V, Kirkwood K, Peck D, Robertson S, Scott-Lodge L, Zeffert S. Telemedicine for clinical psychology in the Highlands of Scotland. J Telemed Telecare. 1999;5(3):157.

17. Lind L, Karlsson D. A system for symptom assessment in advanced palliative home healthcare using digital pens. Inform Health Soc Care. 2004;29(3-4):199-210.

18. Demiris G, Oliver D, Courtney K. Ethical considerations for the utilization of telehealth technologies in home and hospice care by the nursing profession. Nurs Adm Q. 2006;30(1):56-66.

19. McCall K, Keen J, Farrer K, et al. Perceptions of the use of a remote monitoring system in patients receiving palliative care at home. Int $J$ Palliat Nurs. 2008;14(9):426-431.

20. Johnston B, Kidd L, Wengstrom Y, Kearney N. An evaluation of the use of telehealth within palliative care settings across Scotland. Palliat Med. 2011;26(2):152-161. 
21. Maudlin J, Keene J, Kobb R. A road map for the last journey: home telehealth for holistic end-of-life care. Am J Hosp Palliat Care. 2006;23(5):399-403.

22. Watanabe SM, Fairchild A, Pituskin E, Borgersen P, Hanson J, Fassbender K. Improving access to specialist multidisciplinary palliative care consultation for rural cancer patients by videoconferencing: report of a pilot project. Support Care Cancer. 2013;21(4):1201-1207.

23. van Gurp J, Hasselaar J, van Leeuwen E, Hoek P, Vissers K, van Selm M. Connecting with patients and instilling realism in an era of emerging communication possibilities: a review on palliative care communication heading to telecare practice. Patient Educ Couns. 2013;93(3):504-514.

24. Kamal AH, Swetz KM, Dy S, Tien AY, Temel JS, Abernethy AP. Integrating technology into palliative care research. Curr Opin Support Palliat Care. 2012;6(4):525-532.

25. Andre B, Ringdal GI, Loge JH, Rannestad T, Kaasa S. The importance of key personnel and active management for successful implementation of computer-based technology in palliative care: results from a qualitative study. Comput Inform Nurs. 2008;26(4):183-189.

26. Roberts D, Tayler C, MacCormack D, Barwich D. Telenursing in hospice palliative care. Can Nurse. 2007;103(5):24.

27. May C, Harrison R, Finch T, MacFarlane A, Mair F, Wallace P. Understanding the normalization of telemedicine services through qualitative evaluation. BMJ. 2003;10(6):596.

28. May C, Mort M, Williams T, Mair F, Gask L. Health technology assessment in its local contexts: studies of telehealthcare. Soc Sci Med. 2003;57(4):697-710.

29. Fischer S, Stewart T, Mehta S, Wax R, Lapinsky S. Handheld computing in medicine. J Am Med Inform Assoc. 2003;10(2):139.

30. Jerant A, Azari R, Nesbitt T. Reducing the cost of frequent hospital admissions for congestive heart failure: a randomized trial of a home telecare intervention. Med Care. 2001;39(11):1234-1245.

31. Field M, Grigsby J. Telemedicine and remote patient monitoring. JAMA. 2002;288(4):423.

32. Mooney K, Beck S, Friedman R, Farzanfar R. Telephone-linked care for cancer symptom monitoring. Cancer Pract. 2002;10(3):147-154.

33. Kearney N, McCann L, Norrie J, et al. Evaluation of a mobile phonebased, advanced symptom management system (ASyMSC) in the management of chemotherapy-related toxicity. Support Care Cancer. 2009; 17(4):437-444.

34. Regnard C. Using videoconferencing in palliative care. Palliat Med. 2000;14(6):519.

35. Norum J, Jordhoy M. A university oncology department and a remote palliative care unit linked together by email and videoconferencing. J Telemed Telecare. 2006;12(2):92.

36. Coyle N, Khojainova N, Francavilla J, Gonzales G. Audio-visual communication and its use in palliative care. J Pain Symptom Manage. 2002;23(2):171-175

37. Oliver P, Debra R, Demiris G, Porock D. The usability of videophones for seniors and hospice providers: a brief report of two studies. Comput Biol Med. 2005;35(9):782-790.

38. van Gurp J, van Selm M, van Leeuwen E, Hasselaar J. Transmural palliative care by means of teleconsultation: a window of opportunities and new restrictions. BMC Med Ethics. 2013;14(1):12.

39. Young G. Implementing a patient administration system. European Journal of Palliative Care. 2000;7(1):26-28

40. Marie Curie Cancer Care. Telemedicine Supporting Delivering Palliative Care Services and Education In Rural Wales: London: Marie Curie Cancer Care; 2008.

41. Saysell E, Routley C. Telemedicine in community-based palliative care: evaluation of a videolink teleconference project. Int J Palliat Nurs. 2003;9(11):489.

42. Lloyd-Williams M, Rashid A. An analysis of calls to an out-of-hours palliative care advice line. Public Health. 2003;117(2):125-127.

43. Yardley S, Codling J, Roberts D. Experiences of 24-hour advice line services: a framework for good practice and meeting NICE guidelines. Int J Palliat Nurs. 2009;15(6):266.
44. Salt S. Letter to the Editor: What kind of requests do healthcare professionals make of a telephone out of hours specialist palliative care advice service? The experience of one hospice over a year. Palliat Med. 2007;21(1):61

45. Kuziemsky C, Jahnke J, Lau F. The e-hospice: beyond traditional boundaries of palliative care. Telematics and Informatics. 2006;23(2):117-133.

46. Demiris G, Oliver D, Fleming D, Edison K. Hospice staff attitudes towards telehospice. Am J Hosp Palliat Care. 2004;21(5):343.

47. Chochinov H, Stern A. The Canadian Virtual Hospice http://www. virtualhospice.ca. J Palliat Care. 2005;20(1):5-6.

48. Canadian Virtual Hospice [homepage on the Internet]. Available from: http://www.virtualhospice.ca/. Accessed September 9, 2014.

49. ehospice [homepage on the Internet]. Available from: http://www ehospice.com/uk/en-gb/aboutus.aspx. Accessed September 9, 2014.

50. Colvin J, Chenoweth L, Bold M, Harding C. Caregivers of older adults: advantages and disadvantages of internet based social support. Fam Relat. 2004;53(1):49-57.

51. Demiris G, Parker ODR, Courtney KL, Porock D. Use of technology as a support mechanism for caregivers of hospice patients. $J$ Palliat Care. 2005;21(4):303.

52. White MH, Dorman SM. Online support for caregivers: analysis of an Internet Alzheimer mailgroup. Comput Nurs. 2000;18(4):168-180.

53. Johnston BM, Maguire R, Connaghan J, Kotronoulas G, Taylor A, Kearney N. Developing and testing an intervention to monitor symptoms in patients receiving palliative care at home using the mobile phone based remote monitoring Advanced Symptom Management System (Palliative Care). The ASyMSC-P Study. Dundee: University of Dundee; 2011.

54. Thekkumpurath P, Venkateswaran C, Kumar M, Newsham A, Bennett M. Screening for psychological distress in palliative care: performance of touch screen questionnaires compared with semistructured psychiatric interview. J Pain Symptom Manage. 2009;38(4):597-605.

55. Maguire R, Cowie J, Leadbetter C, et al. The development of a side effect risk assessment tool (ASyMSC-SERAT) for use in patients with breast cancer undergoing adjuvant chemotherapy. J Res Nurs. 2009;14(1):27.

56. Bedini R, Belardinelli A, Giansanti D, Guerriero L, Macellari V, Morelli S Quality assessment and cataloguing of telemedicine applications. J Telemed Telecare. 2006;12(4):189.

57. Sealy T, Perry F, Stockdale S. Audit of a specialist palliative care triage advice line within South Tees. BMJ Support Palliat Care. 2014;4:A87-A88.

58. Wye L, Lasseter G, Percival J, Duncan L, Simmonds B, Purdy S. What works in 'real life' to facilitate home deaths and fewer hospital admissions for those at end of life? Results from a realist evaluation of new palliative care services in two English counties. BMC Palliat Care. 2014;13(1):37

59. Campbell C, Harper A, Elliker M. Introducing 'Palcall': an innovative out-of-hours telephone service led by hospice nurses. Int J Palliat Nurs. 2005;11(11):586.

60. Laila M, Rialle V, Nicolas L, Duguay C, Franco A. Videophones for the delivery of home healthcare in oncology. Stud Health Technol Inform. 2008;136:39.

61. Hebert MA, Paquin M-Je, Whitten L, Cai P. Analysis of the suitability of 'video-visits' for palliative home care: implications for practice. J Telemed Telecare. 2007;13(2):74-78.

62. Doolittle GC, Whitten P, Mccartney M, Cook D, Nazir N. An empirical chart analysis of the suitability of telemedicine for hospice visits. Telemed J E Health. 2005;11(1):90-97.

63. Gagnon M-P, Lamothe L, Hebert M, Chanliau J, Fortin J-P. Telehomecare for vulnerable populations: the evaluation of new models of care. Telemed J E Health. 2006;12(3):324-331.

64. Finch T, May C, Mair F, Mort M, Gask L. Integrating service development with evaluation in telehealthcare: an ethnographic study. BMJ. 2003;327(7425):1205.

65. Jennett P, Gagnon M, Brandstadt H. Preparing for success: readiness models for rural telehealth. J Postgrad Med. 2005;51(4):279. 
66. Becker R. Can the palliative care services of today keep up and match the expectations of the 'baby boomer' generation? Int J Palliat Nurs. 2009;15(3):108
67. Brigden M, Minty A, Pilatzke S, della Vedova L, Sherrington L, McPhail K. Strengths and weaknesses of teleoncology. Oncology Exchange. $2008 ; 7: 8-12$.

\section{Publish your work in this journal}

Smart Homecare Technology and TeleHealth is an international, peer-reviewed, open access online journal publishing original research, reviews, editorials and commentaries on the application of technology to support people and patients at home and in assisted living centers to optimize healthcare and management resources. Specific topics in the journal include: Development and application of devices within the home and embedded in appliances; Healthcare provider communication and education tools; and drug ordering and adherence. The manuscript management system is completely online and includes a very quick and fair peer-review system, which is all easy to use. Visit http://www.dovepress.com/ testimonials.php to read real quotes from published authors.

Submit your manuscript here: http://www.dovepress.com/smart-homecare-technology-and-telehealth-journal 